\title{
PSTPIP2 dysregulation contributes to aberrant terminal differentiation in GATA-1-deficient megakaryocytes by activating LYN
}

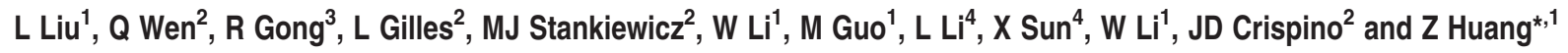

GATA1 mutations are tightly associated with transient myeloproliferative disorder (TMD) and acute megakaryoblstic leukemia (AMKL) in children with Down syndrome. Numerous genes are altered in GATA-1-deficient megakaryocytes, which may contribute to the hyperproliferation and abnormal terminal differentiation of these malignant cells. In this study, we demonstrate that Pstpip2 is a GATA-1-repressed gene that controls megakaryopoiesis. Ectopic expression of PSTPIP2 impaired megakaryocytic differentiation as evidenced by a decrease of CD41 expression and reduced DNA content in K562 cells. PSTPIP2 overexpression also caused enhanced activation of Src family kinases and subsequently reduced ERK phosphorylation. Consistently, PSTPIP2 knockdown showed the opposite effect on differentiation and signaling. Moreover, the W232A mutant of PSTPIP2, defective in its interaction with PEST family phosphatases that recruit c-Src terminal kinase (CSK) to suppress Src family kinases, failed to inhibit differentiation and lost its ability to enhance Src family kinases or reduce ERK phosphorylation. In fact, the W232A mutant of PSTPIP2 promoted megakaryocyte differentiation. These observations suggest that PSTPIP2 recruiting PEST phosphatases somehow blocked CSK activity and led to enhanced activation of Src family kinases and reduced ERK phosphorylation, which ultimately repressed megakaryocyte differentiation. Supporting this idea, PSTPIP2 interacted with LYN and the expression of a dominant negative LYN (LYN DN) overwhelmed the inhibitory effect of PSTPIP2 on differentiation and ERK signaling. In addition, a constitutively active LYN (LYN CA) normalized the enhanced megakaryocyte differentiation and repressed ERK signaling in PSTPIP2 knockdown cells. Finally, we found that PSTPIP2 repressed ERK signaling, differentiation, and proliferation and verified that PSTPIP2 upregulation repressed megakaryocyte development in primary mouse bone marrow cells. Our study thus reveals a novel mechanism by which dysregulation of PSTPIP2 due to GATA-1 deficiency may contribute to abnormal megakaryocyte proliferation and differentiation in pathogenesis of related diseases.

Cell Death and Disease (2014) 5, e988; doi:10.1038/cddis.2013.512; published online 9 January 2014

Subject Category: Experimental Medicine

Extrinsic thrombopoietin (TPO) stimulation and intrinsic GATA-1 expression are two critical driving forces that regulate distinct features of megakaryopoiesis. TPO stimulation activates three major pathways including JAK/STATs, MAPK/ERK, and PI3K/AKT, which promote cell proliferation and eventually drives progenitor cells to commit into megakaryocyte lineage. TPO-null or its receptor MPL-deficient mice develop thrombocytopenia with immature megakaryocytes. ${ }^{1,2}$ On the other hand, GATA-1 deficiency leads to hyperproliferation of megakaryocytes that fail to undergo terminal differentiation or give rise to platelets. ${ }^{3-5}$ Mice with loss of GATA-1, specifically in megakaryocytes, develop thrombocytopenia and myelofibrosis. ${ }^{6}$ In humans, GATA1 mutations are tightly associated with acute megakaryoblastic leukemia in children with Down syndrome (DS-AMKL) and lead to production of a N-terminus truncated form of GATA-1 (GATA-1s). ${ }^{7,8}$ GATA-1s knock-in mice display transient expansion of megakaryocytes in the fetus and mimic human transient myeloproliferative disorder (TMD) in Down syndrome neonates. ${ }^{9}$ Nevertheless, how GATA-1 target genes may coordinate with TPO signaling and contribute to megakaryocyte hyperproliferation and abnormal terminal differentiation in the pathogenesis of related diseases has not been fully addressed.

Several cytokine signaling components have been shown to be GATA-1 target genes. For instance, JAK2 has been found to be significantly downregulated in GATA-1low megakaryocytes that display reduced TPO signaling with

\footnotetext{
${ }^{1}$ College of Life Sciences, Wuhan University, Wuhan, Hubei, China; ${ }^{2}$ Department of Medicine, Division of Hematology and Oncology, Northwestern University Feinberg School of Medicine, Chicago, IL, USA; ${ }^{3}$ Hubei International Travel Healthcare Center, Hubei Entry-Exit Inspection and Quarantine Bureau of P. R. China, Wuhan, Hubei, China and ${ }^{4}$ Department of Hematology, Jiangsu Province Hospital of TCM, Nanjing University of Chinese Medicine, Nanjing, Jiangsu, China

${ }^{*}$ Corresponding author: Z Huang, College of Life Sciences, Wuhan University, Wuhan, Hubei 430072, China. Tel: +86 27 68756660; Fax: +86 27 68756660; E-mail: Z-huang@whu.edu.cn

Keywords: TPO signaling; GATA-1; PSTPIP2; megakaryopoiesis; LYN

Abbreviations: PSTPIP2, proline-serine-threonine phosphatase-interacting protein 2; 5-FU, 5'-fluouracil; AMKL, acute megakaryoblastic leukemia; CA, constitutively active; CFU-GM, colony-forming unit-granulocyte/macrophage; CFU-MK, colony-forming unit-megakaryocytic; CTH, carboxyl-terminal homology; WASP, WiskottAldrich syndrome protein; CSF-1, colony-stimulating factor-1; CSK, C-terminal Src kinase; DN, dominant negative; DS-AMKL, Down syndrome acute megakaryoblastic leukemia; TPO, thrombopoietin; TMD, transient myeloproliferative disorder; MNC, mononuclear cell; SFK, Src family kinase; WT, wild type

Received 25.6.13; revised 28.10.13; accepted 29.10.13; Edited by A Stephanou
} 
low STAT3 and STAT5 phosphorylation. ${ }^{10-12}$ In addition, reduced STAT1 and interferon-gamma (IFN- $\gamma$ ) receptor expression also suggests a possible involvement of IFN- $\gamma$ signaling in megakaryopoiesis. Indeed, recent research has revealed an important role of IFN- $\gamma /$ STAT1 signaling in megakaryocyte development in essential thrombocythemia. ${ }^{12,13}$ These observations strongly suggest that GATA-1 may participate in cytokine signaling, including TPO and IFN$\gamma$, that have an important role in both physiological and pathological megakaryopoiesis. Nevertheless, how GATA-1 may regulate cytokine signaling remained to be further addressed.

PSTPIP2 (proline-serine-threonine phosphatase-interacting protein 2) has been suggested to be a direct GATA1 target gene in megakaryocytes. Upregulation of PSTPIP2 was observed in GATA-1low or GATA-1s megakaryocytes. $^{9,11}$ Recent ChIP-Seq study further revealed a GATA-1-binding site in the intron 1 region of this gene locus. ${ }^{14-16}$ PSTPIP2 belongs to a family that contains a conserved Fes CIP4 homology $(\mathrm{FCH})$ domain in $\mathrm{N}$ terminal. Compared with PSTPIP1, PSTPIP2 lacks the SH3 domain that is necessary for interaction with the Wiskott-Aldrich syndrome protein (WASP). Instead, it binds to the CTH (carboxyl-terminal homology) region of PEST family phosphatases. ${ }^{17}$ PSTPIP2 is tyrosine-phosphorylated on colony-stimulating factor-1 (CSF-1) treatment. ${ }^{17}$ It is also efficiently phosphorylated after $\mathrm{V}$-Src transfection. ${ }^{18}$ In mouse models, PSTPIP2 deficiency causes autoinflammatory disease involving extramedullary hematopoiesis, as evidenced by expansion of macrophage progenitors. These mice also exhibit skin and bone lesion and mimicking human multiple osteomyelitis. ${ }^{19,20}$ Mechanistic studies showed that Pstpip2 deficiency led to an increased responsiveness to CSF-1 stimuli leading to a hyperactivation of Erk1/2 and STAT1 in mature macrophages. ${ }^{19}$ Thus, PSTPIP2 acts as a negative feedback regulator of CSF-1R signaling to suppress inflammation and osteoclastogenesis. Considering the dysregulation pattern of PSTPIP2 in GATA-1-deficient megakaryocytes, PSTPIP2 may contribute to abnormal megakaryocyte differentiation in this setting.

In this study, we probe the function of PSTPIP2 in megakaryocyte differentiation. Our study demonstrates that Pstpip2 is a GATA-1 target gene and that it inhibits megakaryocyte differentiation by repressing ERK activating through recruiting PEST phosphatases and activating LYN. Thus, we reveal a novel mechanism by which GATA-1 secures TPO signaling-induced ERK activation to ensure megakaryocyte differentiation through repression of the negative regulator PSTPIP2 in normal megakaryopoiesis. Dysregulation of PSTPIP2 due to GATA-1 deficiency may contribute to abnormal megakaryocyte terminal differentiation in the pathogenesis of the related diseases.

\section{Results}

PSTPIP2 is a GATA-1 target gene and upregulated on megakaryocytic differentiation in K562 cells. Pstpip2 was reported to have a role in monocytes/macrophages maturation. ${ }^{19,20}$ We verified that Pstpip2 expression was relatively high in mouse whole bone marrow cells or bone marrow from 5'-fluouracil-treated mice (5-FU-treated) as compared with spleen or lymph node cells (Supplementary Figure S1). We also observed high expression level of PSTPIP2 in mononuclear cells (MNCs) isolated from human cord blood as compared with CMK, K562, and HL-60 cells (Supplementary Figure S1). Pstpip2 was suggested to be a GATA-1 target gene and a putative GATA-1-binding site was identified in the first intron. ${ }^{14-16}$ Thus, we tested the response of Pstpip2 gene promoter to GATA-1 using a luciferase reporter system. A 1-kb fragment of promoter upstream region of the Pstpip2 gene was subcloned into pGL3 Basic vector (termed as P2-pro). A second vector was made by subcloning the 265-bp core region of the putative GATA-1-binding site between the P2-pro and luciferase (termed as P2-pro/G1BS). We found that GATA-1 failed to repress P2-pro activity. In contrast, GATA-1 significantly inhibited P2-pro/G1BS activity (Figure 1a). These results suggest that the putative GATA-1-binding site in the intron 1 region of Pstpip2 gene may mediate inhibitory effect on its promoter. Furthermore, we overexpressed GATA-1 in GATA-1-null G1ME cells. We found that the mRNA level of Pstpip2 was significantly reduced by GATA-1 (Figure 1b). To further demonstrate that Pstpip2 is a direct GATA-1 target gene, we performed GATA-1 chromatin immunoprecipitation (ChIP) in $\mathrm{Y} 10$ cells and measured DNA amount by quantitative PCR. We found that The chromatin DNA of putative GATA-1-binding site in the intron 1 region of Pstpip2 gene was significantly enriched by almost sixfold in GATA-1 antibody group compared with that in the normal goat IgG control antibody group (Figure 1c). In contrast, the amount of DNA from the negative control region in Gapdh gene promoter was comparable in both groups. These observations verify that Pstpip2 is an authentic GATA-1 target gene.

To probe how PSTPIP2 expression may correlate to megakaryocyte differentiation, we measured its expression in K562 cells undergoing TPA-induced megakaryocytic differentiation. TPA treatment induced robust megakaryocyte differentiation evidenced by increased expression of CD41 (Figure 1d). We observed a significant upregulation of PSTPIP2 both at mRNA and protein levels, whereas GATA1 protein was coincidently downregulated (Figure 1e). To probe how PSTPIP2 may be regulated in megakaryocyte differentiation, we cultured megakaryocytes from human CD34 + cells up to 10 days and measured GATA1 and PSTPIP2 expression. We observed constant upregulation of GATA1 over time as expected (Figure 1f). Unexpectedly, PSTPIP2 expression did not seem to reversely correlate to GATA-1 expression: the PSTPIP2 expression was also upregulated over time (Figure 1f). We reasoned that PSTPIP2 upregulation in megakaryocyte differentiation was restricted by GATA-1 and PSTPIP2 upregulation would be more significant in GATA-1-deficient megakaryocytes. Therefore, we detected Pstpip2 expression in GATA-1low megakaryocytes and compared with that of WT. We discovered that Pstpip2 expression was dramatically upregulated in GATA1low megakaryocytes, which show impaired differentiation (Figure 1g). All these observations suggest a potential correlation among PSTPIP2, GATA-1, and megakaryocyte differentiation. 

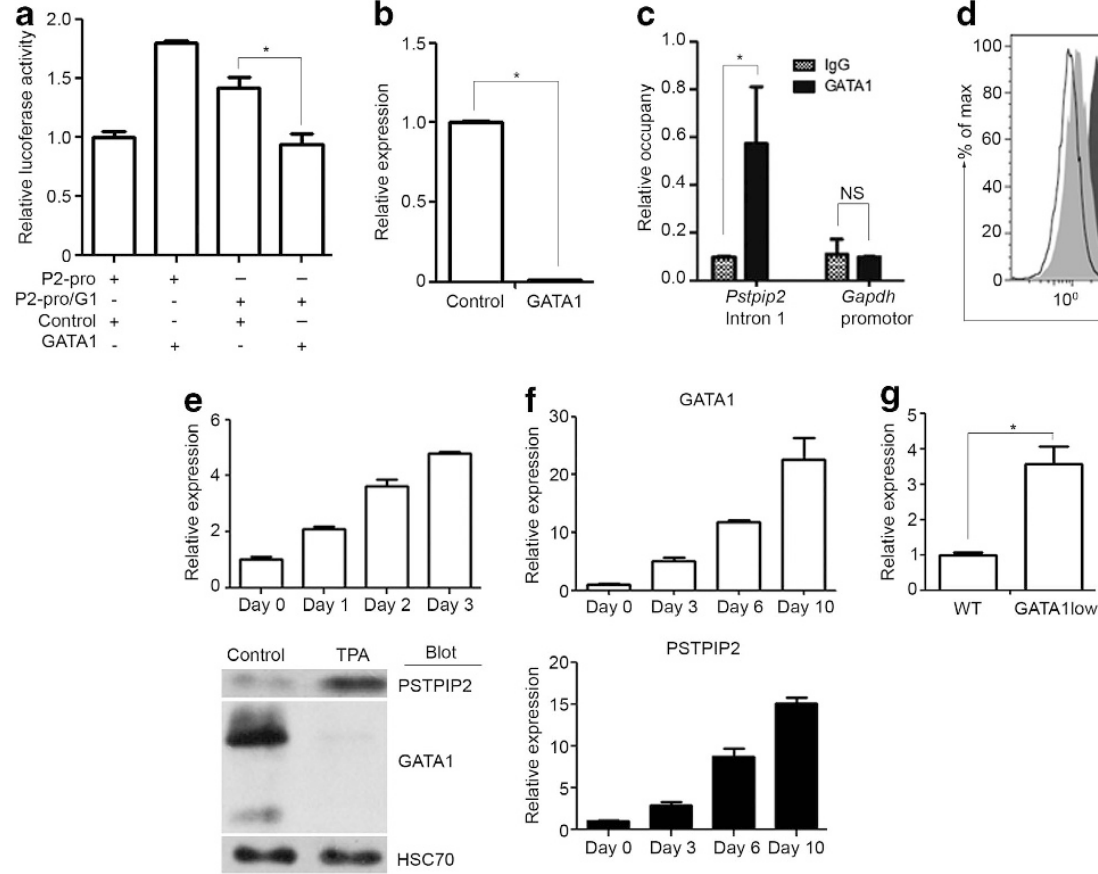

Figure 1 Upregulation of PSTPIP2 in megakaryocytes. (a) Pstpip2 promoter (P2-pro) or Pstpip2 promoter plus GATA-1-binding site (P2-pro/G1BS) luciferase constructs were cotransfected with control or GATA-1 expression vector into 293 T cells as indicated. The luciferase activity in each sample was measured and presented as relative luciferase activity. (b) G1ME cells were infected with control or GATA-1 expression vector. Both constructs carried puromycin-resistant gene. The infected cell were selected with puromycin for 3 days and harvested for quantitative RT-PCR to measure Pstpip2 expression. (c) GATA-1 was immunoprecipitated with an anti-GATA-1 antibody in Y10 cells. The chromatin that was co-immunoprecipitated with GATA-1 antibody or normal goat lgG (IgG) was purified. The DNA quantitation of Pstpip2 intron 1 region or Gapdh promoter was measured by quantitative PCR. (d) K562 cells were treated with or without TPA for days as indicated. The CD41 expression of the resultant cells was measured by staining cells with an anti-CD41-PE antibody and analyzed by flow cytometry. (e) K562 cells treated with TPA (TPA) or without TPA (Control) for 3 days were collected to extract total RNA and protein. The mRNA expression level of Pstpip2 (top panel) was measured by quantitative RT-PCR. The protein expression level of PSTPIP2 or GATA-1 (the bottom panel) was also measured by western blot. HSC 70 was used as a loading control. (f) Human CD34 + cells were cultured in the presence of $10 \mathrm{ng} / \mathrm{ml}$ TPO for different times as indicated. The expression of GATA1 and PSTPIP2 over time was measured by quantitative RT-PCR. (g) Lineage-depleted bone marrow progenitor cells from WT or GATA-1low mouse were cultured in the presence of TPO for 3 days. The megakaryocytes were purified through a BSA gradient column. The expression of Pstpip2 was detected by quantitative RT-PCR. * Indicates significance $(P<0.05)$; NS indicates non-significance $(P>0.05)$ compared with control

PSTPIP2 negatively regulates TPA-induced megakaryocyte differentiation. The expression pattern of PSTPIP2 in K562 cells undergoing megakaryocytic differentiation suggests a potential role of PSTPIP2 in this process. To test this possibility, we overexpressed PSTPIP2 and checked its effect on megakaryocyte differentiation (Figure 2a). PSTPIP2 significantly reduced CD41 expression compared with control cells (Figure 2b). The expression of other megakaryocyte differentiation marker CD61 was also significantly downregulated by PSTPIP2 (data not shown). For consistency, we used CD41 as the differentiation marker throughout this study. PSTPIP2 also reproducibly decreased the percentage of cell population with DNA content greater than $4 \mathrm{~N}$ (Figure $2 \mathrm{c}$ ). In addition, we knocked PSTPIP2 down by shRNA technique (shPSTPIP2) and observed an opposite phenotype (Figures $2 \mathrm{~d}-\mathrm{f}$ ). To exclude the off-target effect of shRNA, we designed a second shRNA to knock-down PSTPIP2 and observed similar effect (Supplementary Figure S2). These results suggest that PSTPIP2 dysregulation inhibits megakaryocyte differentiation. We performed overexpression or downregulation of PSTPIP2 in CMK cells and observed similar effect of PSTPIP2 on megakaryocyte differentiation (data not shown).
PEST family phosphatases are required for the effect of PSTPIP2 on megakaryocyte proliferation and differentiation. PSTPIP2 interacted with PEST family phosphatases that mediated suppression of Src family kinases by recruiting c-Src kinase (CSK). ${ }^{18,21}$ Src family kinases in turn phosphorylate PSTPIP2 and inhibit ERK. ${ }^{22}$ To test whether PEST family phosphatases or PSTPIP2 tyrosine phosphorylation by Src family kinases are required for its function, we created two mutant forms of PSTPIP2. Two Src family kinases tyrosine phosphorylation sites $\mathrm{Y} 323$ and $\mathrm{Y} 333$ are substituted with phenylalanine in $Y 323 / 333 F$ mutation, whereas the W232A mutation has a substitution of tryptophan W232 by phenylalanine and loses the interaction of PSTPIP2 with PEST family phosphatases. As expected, wild-type PSTPIP2 effectively repressed megakaryocyte differentiation, as evidenced by reduced CD41 expression and DNA content (Figures $3 a$ and b). Y323/333F mutant retained its capacity to repress CD41 expression but lost its ability to reduce DNA content compared with wild-type PSTPIP2, suggesting that tyrosine phosphorylation by Src family kinases partially contributes to PSTPIP2 function. In contrast, W232A mutant completely lost its ability to impair megakaryocyte differentiation. In fact, it exhibited the opposite effect: it promoted CD41 expression and increased DNA content in K562 cells 

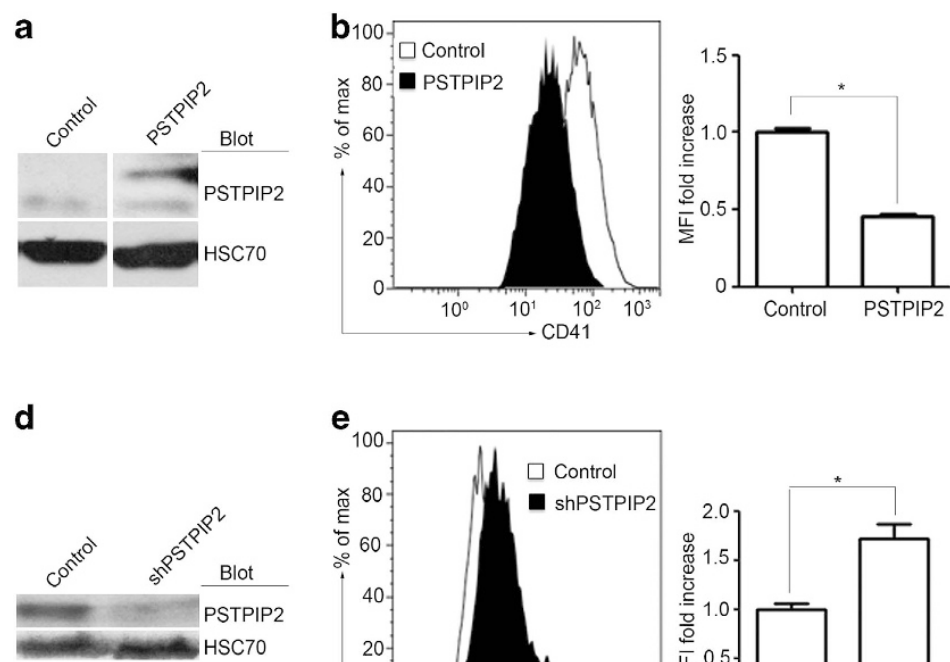
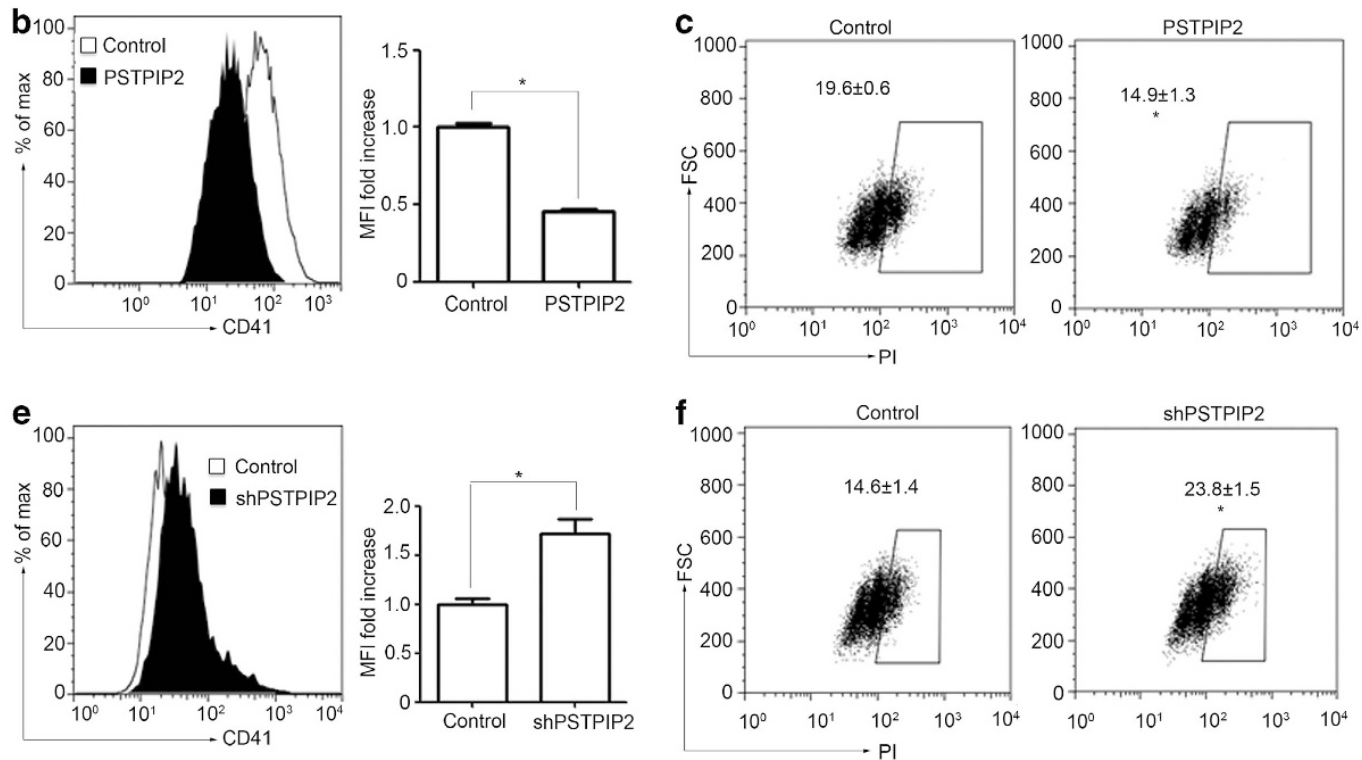

Figure 2 PSTPIP2 negatively regulates megakaryocytic differentiation in K562 cells. (a) Control vector or PSTPIP2-overexpressing vector was introduced into K562 cells through lentiviral infection. The endogenous and exogenous (FLAG-tagged) PSTPIP2 expression levels were detected by western blot with an anti-PSTPIP2 antibody. The repositioned lanes were from the same blot. (b) Control cells or PSTPIP2-overexpressing cells were treated with TPA (10 nM) for 3 days. The CD41 expression of the resultant cells was measured by staining cells with an anti-CD41-PE antibody and analyzed by flow cytometry. The bar graph is the statistics of the left panel. (c) The TPA-treated cells were fixed, permeabilized, and stained with PI. The DNA content of TPA-treated cells was analyzed by flow cytometry. Gated events represent the cells with DNA content greater than 4 N. Numbers (mean \pm S.D.) indicate percentage of the gated cells. Results are statistics of two independent experiments. (d) Control vector or a vector expressing shRNA specific for human PSTPIP2 (shPSTPIP2) were introduced into K562 cells through lentiviral infection. The protein expression level of PSTPIP2 was detected by western blot. (e) Control or shPSTPIP2 cells were treated with TPA for 2 days. The CD41 expression of the resultant cells was measured by staining cells with an anti-CD41-PE antibody and analyzed by flow cytometry. The bar graph is the statistics of the left panel. (f) TPA-treated cells were also used for measuring DNA content by PI staining and analyzed by flow cytometry. Numbers (mean \pm S.D.) indicate percentage of the gated cells with DNA content greater than $4 \mathrm{~N}$. Results are statistics of two independent experiments. ${ }^{*}$ Indicates $P<0.05$ compared with control

(Figures $3 a$ and $b$ ). These results suggest that PEST family phosphatases are required for megakaryocyte differentiation inhibition by PSTPIP2.

PSTPIP2 interaction with the PEST family phosphatases may affect the downstream signaling. Thus, we examined the activation of Src family kinases and ERK. We used an antibody that specifically recognizes phosphorylation of Src at tyrosine 416 ( $p$-Src Y416) that enhances enzyme activity and can serve as an activation marker of Src family kinases. This antibody also cross-reacts with other Src family kinase members (Lyn, Fyn, Lck, Yes, and Hck) when tyrosine phosphorylation occurred at equivalent sites, which allowed us to detect the activity of total Src family kinases. Interestingly, with TPA treatment wild-type PSTPIP2 (WT) increased p-Src Y416 and reduced ERK phosphorylation. Y323/333F retained the ability to increase Src family kinases phosphorylation but repressed ERK phosphorylation (p-ERK) to a much less extent compared with that of WT PSTPIP2. In contrast, W232A failed to enhance p-Src Y416 and did not alter ERK phosphorylation at all (Figure $3 \mathrm{c}$ ). Further supporting the effect of PSTPIP2 on signaling, we observed the reverse phenotype in PSTPIP2 knockdown cells: PSTPIP2 downregulation led to reduced $p$-Src Y416 and increased p-ERK (Figure 3d). Considering ERK phosphorylation has a critical role in megakaryocyte differentiation, these results suggest that PSTPIP2 inhibit megakaryocyte differentiation by suppressing ERK through Src family kinases.
LYN and FYN are two major Src family kinases that are activated in TPO-induced megakaryocyte development. ${ }^{22}$ To confirm which Src family kinase is recruited by PSTPIP2, we performed co-immunoprecipitation with an antibody specific for FLAG that was tagged to PSTPIP2. Noticeably, we found that LYN but not FYN, was co-immunoprecipitated with WT PSTPIP2 (Figure 3e). This interaction was not affected by tyrosine phosphorylation of PSTPIP2 and was not dependent on PEST family phosphatase, as Y323/333F and W232A mutants showed similar interactions as WT PSTPIP2 (Figure $3 e$ ). These observations suggest that LYN is the major Src family kinase that is recruited by PSTPIP2 to repress ERK phosphorylation and mediate suppression of megakaryocyte differentiation. We also found that $\mathrm{SHC}$ was co-immunoprecipitated by WT and Y323/333F PSTPIP2 but not by W232A (Figure 3e). This is consistent with previous findings showing PEST phosphatases interaction with SHC. ${ }^{23}$ Considering that PTP-PEST and PTP-HSCF interact with SHC, we infer that PTP-PEST and PTP-HSCF might be two major PEST family phosphatases involving with PSTPIP2 function in megakaryocyte differentiation.

Taken together, these observations suggest that PSTPIP2 may repress megakaryocyte differentiation by inhibiting ERK through LYN.

PSTPIP2 negatively regulates CD41 expression, MAPK/ ERK signaling, and proliferation in G1ME cells. TPO signaling is the major physiological pathway that promotes 
a
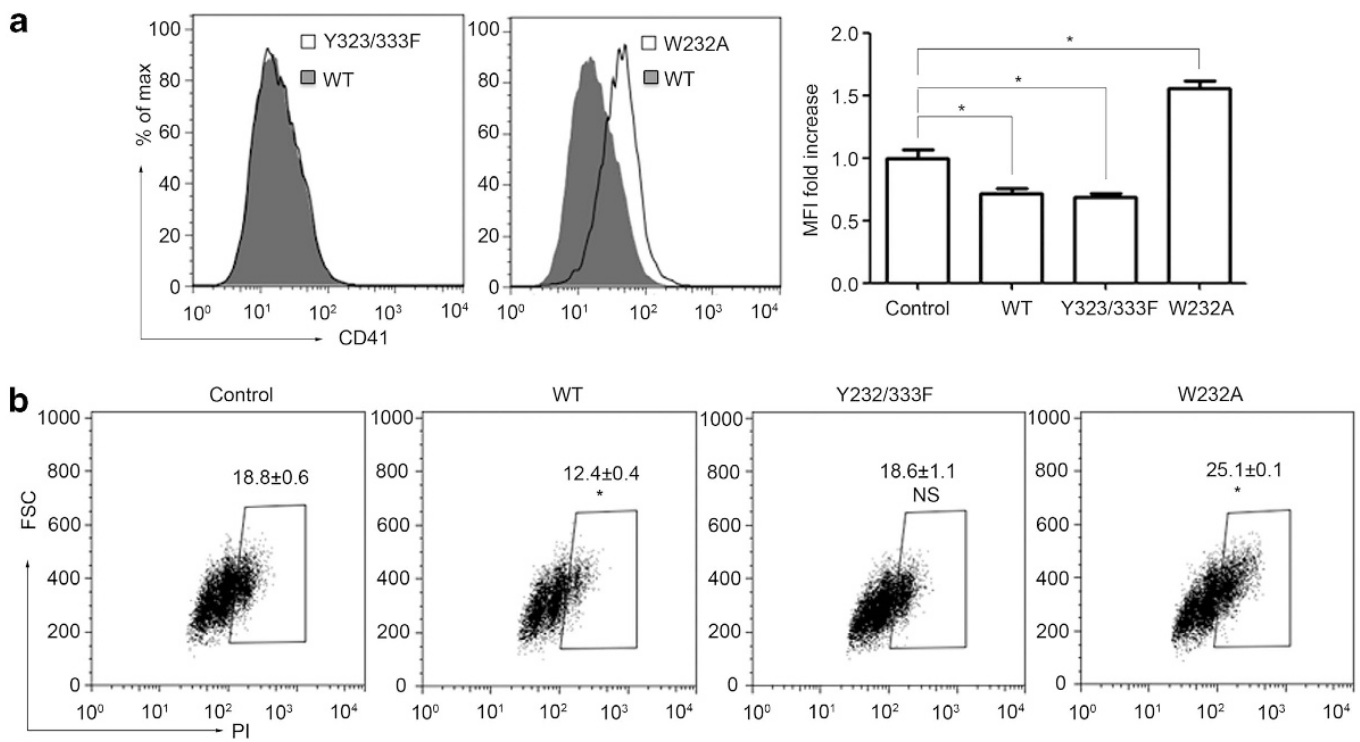

C

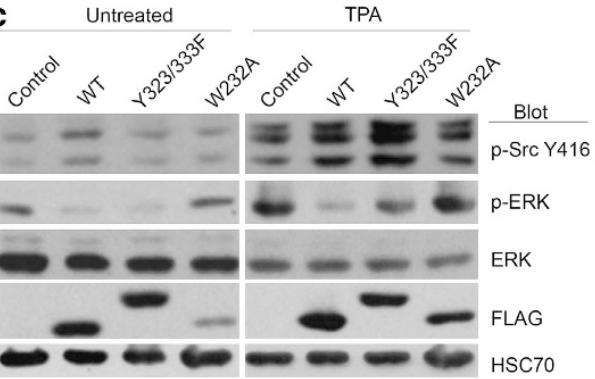

d

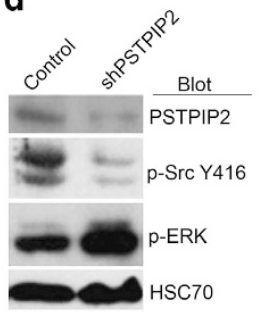

e Input

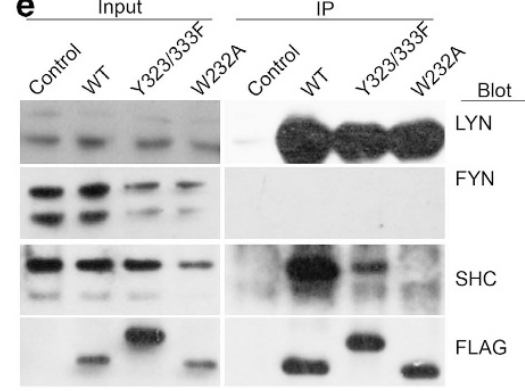

Figure 3 The effect of PSTPIP2 on differentiation and signaling requires PEST family phosphatases. (a) Control vector or vectors expressing wild-type PSTPIP2 (WT), tyrosine sites mutant PSTPIP2 (Y323/333F), or mutant PSTPIP2 that eliminates interaction with PEST family phosphatases (W232A) were introduced into K562 cells by lentiviral infection. The resultant cells were treated with TPA for 2 days. The CD41 expression of the resultant cells was measured by staining cells with an anti-CD41-PE antibody and analyzed by flow cytometry. The bar graph is the statistics of the left panel. (b) TPA-treated cells were used for measuring DNA content by PI-staining cells and analyzed by flow cytometry. Numbers (mean \pm S.D.) indicate the percentage of the gated cells with DNA content greater than $4 \mathrm{~N}$. * Indicates significance $(P<0.05)$; NS indicates non-significance $(P>0.05)$ compared with control. (c) Proteins from TPA-treated or untreated cells were assayed by western blot to detect activation of Src ( $p$-Src Y416) and ERK (p-ERK). The total amounts of ERK and PSTPIP2 variants (FLAG tagged; Y323/333F were double tagged with MYC and FLAG) were also measured. HSC 70 served as a loading control. The repositioned lanes were from the same blot. (d) Proteins from control or shPSTPIP2 cells were used for western blot to detect the activation of Src (p-Src Y416) and ERK (p-ERK). (e) Proteins from control, WT, Y323/333F, or W232A cells were used for immunoprecipitation with FLAG antibody. The amount of FYN, LYN, and SHC that were co-immunoprecipitated by FLAG antibody was measured by western blot. FLAG blot demonstrated the same amount of the PSTPIP2 variants was loaded for measuring. The repositioned lanes were from the same blot

megakaryopoiesis. Thus, we next used a TPO-dependent, GATA-1-null megakaryocyte cell line, G1ME cells, to test the function of PSTPIP2 in megakaryocyte differentiation under more physiological relevance. Reconstitution with full-length GATA-1 induces megakaryocytic terminal differentiation in the presence of TPO. ${ }^{24}$ We found that PSTPIP2 overexpression reduced CD41 expression, increased p-Src Y416, and decreased ERK activation (Figures $4 a$ and b). These findings mirrored the phenotype of PSTPIP2 in K562 cells. Interestingly, we noticed that PSTPIP2 overexpression also effectively repressed G1ME cell proliferation. The relative percentage of PSTPIP2-overexpressing cells, monitored by GFP expression, decreased to less than 0.5 within 3 days (Figure 4c).

To dissect how the downstream signaling may possibly contribute to the effect of PSTPIP2 on differentiation and proliferation, we treated G1ME cells with the MAPK/ERK inhibitor Sorafenib and PD98059. Consistent with our findings, inhibition of MAPK/ERK by Sorafenib reduced the percentage of CD41-positive cells. However, both Sorafenib and PD98059 failed to repress cell proliferation (Figures 4d and e). In addition, the Src family kinase inhibitor I (SFK inhibitor) slightly but reproducibly increased CD41. More importantly, SFK inhibition did not cause repression of cell proliferation (Figures $4 \mathrm{f}$ and $\mathrm{g}$ ). Although the statistics did not show significance in control and SFK inhibitor-treated G1ME cells, we believe that this is due to the nature that the G1ME cells express high level of CD41. One can imagine that it is difficult to further significantly boos CD41 expression at such expression level. These observations suggest that the effect of PSTPIP2 on differentiation and proliferation can be uncoupled. MAPK/ERK are indispensable for inhibition of TPO-induced differentiation but not required for proliferation inhibition by PSTPIP2.

To further explore proliferation suppression by PSTPIP2, we measured cell death by Annexin $V$ staining. We did not 

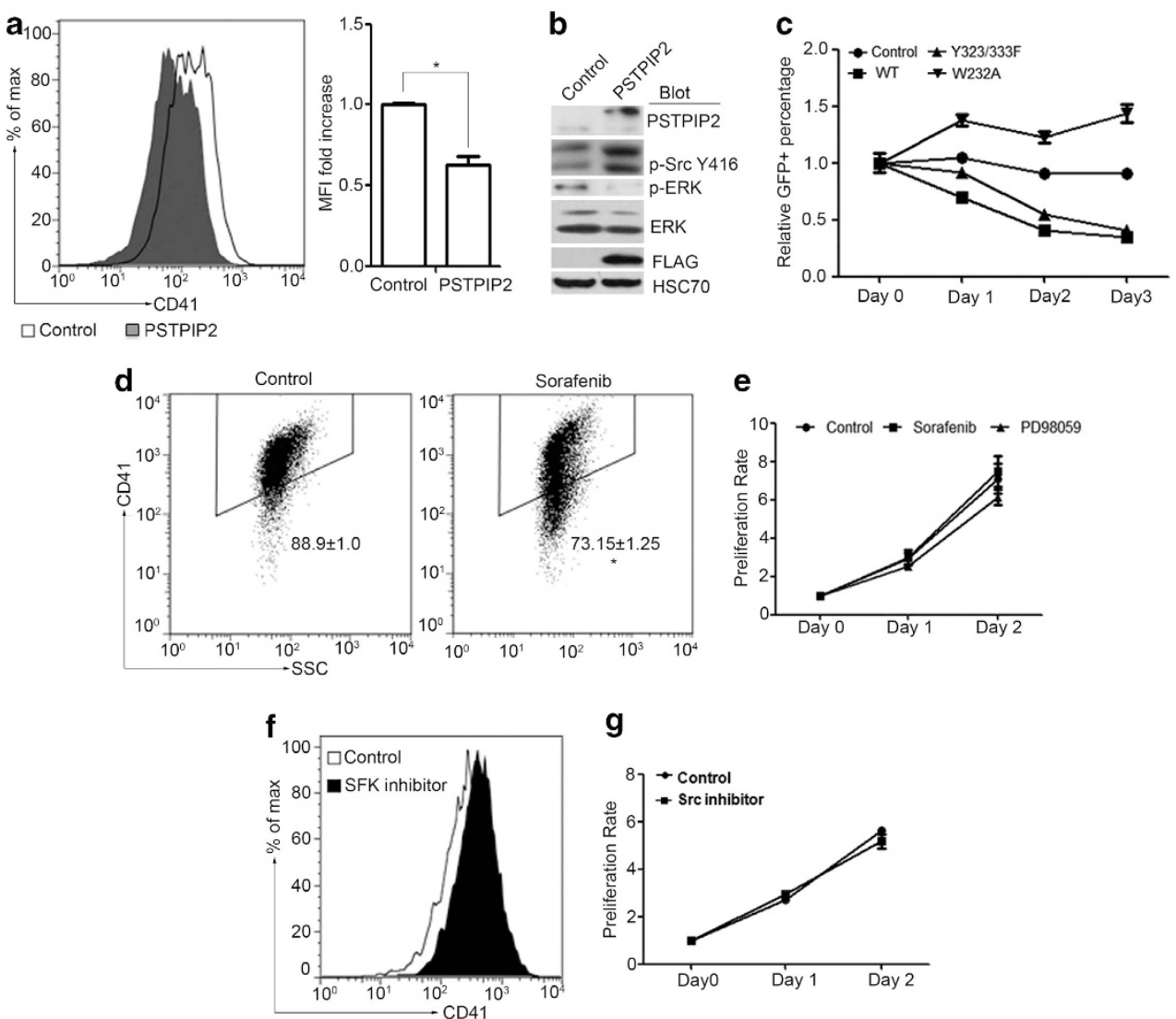

g

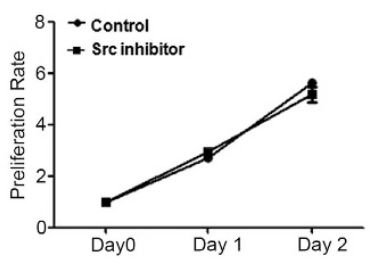

Figure 4 PSTPIP2 represses CD41 expression and proliferation in G1ME cells. (a) Control or PSTPIP2-expressing vectors were introduced into G1ME cells through retroviral infection. The infected cells were selected with puromycin for 2 days and used for detecting CD41 expression by staining cells with anti-CD41-APC antibody and analyzed by flow cytometry. Histogram is representative results of two experiments with similar results. The bar graph is the statistics of the left panel. (b) The infected cells were also harvested to make protein lysates for western blot to detect activation of Src ( $p$-Src Y416) and ERK ( $p$-ERK). The expression of PSTPIP2 (FLAG-tagged) and ERK was also measured. (c) G1ME cells were infected with control vector or vectors expressing WT, Y323/333F, W232A forms of PSTPIP2 and further cultured for 4 consecutive days. All vectors expressed a GFP bicistronically and the percentage GFP + cells on each day were monitored by flow cytometry. The percentage of GFP + cells on each day was normalized to Day 0 ( $24 \mathrm{~h}$ after the last infection) and presented as relative GFP + percentage. Data were statistics of two experiments with similar results. (d) The G1ME cells were cultured with or without Sorafenib $(5 \mu \mathrm{M})$ overnight and the expression of CD41 were detected by staining cells with anti-CD41-APC antibody and analyzed by flow cytometry. Numbers (mean \pm S.D.) indicate percentage of the gated cells. (e) G1ME cells were treated with or without Sorafenib and PD98059 (20 $\mu$ M) for 2 days. The cell density was monitored through counting live cells number under microscopy, normalized to Day 0 (the day seeding the cells), and presented as proliferation rate. Data were statistics of two experiments with similar results. (f) G1ME cells were cultured with or without Src family kinase inhibitor I (SFK inhibitor, $0.1 \mathrm{mM}$ ) overnight and the expression of CD41 was detected by staining cells with anti-CD41-APC antibody and analyzed by flow cytometry. Histogram was representative results of two experiments with similar results. (g) G1ME cells treated with or without SFK inhibitor for 3 days. The cell proliferation was monitored each day, normalized to original seeding cell density, and presented as proliferation rate. Data were statistics of two experiments with similar results. * Indicates significance $(P<0.05)$ compared with control

observe increased Annexin $V$ staining in PSTPIP2-overexpressing cells compared with that of control cells (Figure 5a). Paradoxically, PI staining revealed an increased cell population in $\mathrm{S}$ phase, which normally suggests better cell proliferation, in PSTPIP2-overexpressing cells (Figure 5b). To solve this problem, we further profiled cell cycle by BrdUlabeling experiment. The percentage of cells at $S$ phase with positive BrdU staining representing cells that undergo DNA replication was dramatically decreased by PSTPIP2 overexpression $\left(33.5 \% \mathrm{BrdU}^{+}\right.$cells in Control versus $6.3 \%$ $\mathrm{BrdU}^{+}$cells in PSTPIP2 overexpression cells) (Figure $5 \mathrm{c}$ ). Impressively, a new cell population in BrdU-staining negative cells between $\mathrm{G} 1$ and $\mathrm{G} 2 / \mathrm{M}$ phase cell populations appeared in PSTPIP2-overexpressing cells $(4.2 \%$ in Control versus $35.9 \%$ in PSTPIP2-overexpressing cells) (Figure 4c). This cell population was comprised of cells in $S$ phase that failed to synthesize DNA on PSTPIP2 overexpression, that is, cell cycle arrest in S phase. Consistent with the phenotype, we detected significant upregulation of two cell cycle regulators p21 and p27 in PSTPIP2-overexpressing cells compared with that of control cells (Figure $5 d$ ). Such proliferation inhibition by PSTPIP2 appeared to be dependent on PEST family phosphatases, as the W232A mutant did not cause a decline in the relative GFP + percentage over time (Figure 4c).

LYN mediates the effect of PSTPIP2 on megakaryocytic differentiation and signaling. To further address the function of Src family kinases in the effect of PSTPIP2 on differentiation and signaling, we inhibited Src family kinase activity by overexpressing a dominant negative LYN (LYN DN) in PSTPIP2-overexpressing K562 cells. Overexpression of LYN DN drastically increased CD41 expression, which overwhelmed the inhibitory effect of PSTPIP2 (Figure 6a). Consistently, LYN DN also normalized the enhanced p-Src 
a
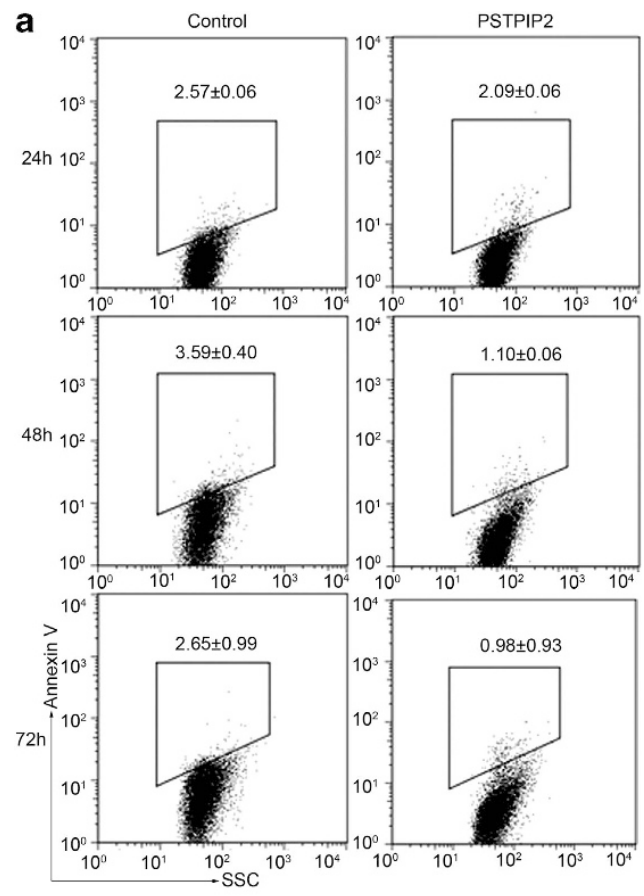

b

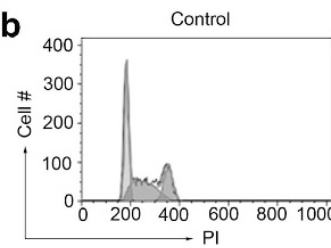

PSTPIP2
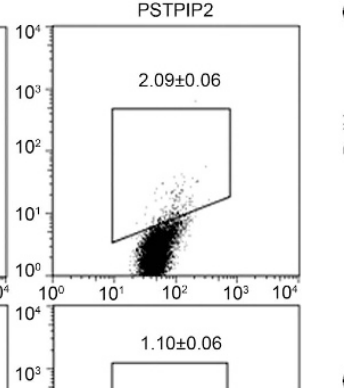

C

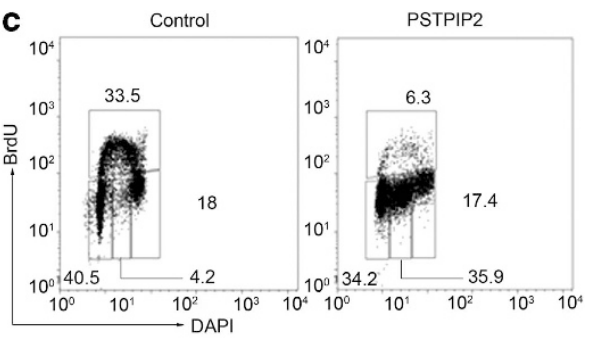

d
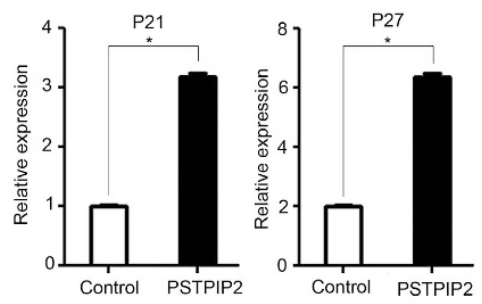

Figure 5 PSTPIP2 causes cell cycle arrest at S phase in G1ME cells. (a) G1ME cells infected with control or PSTPIP2-overexpressing retroviral vectors were stained with PE-labeled Annexin V antibody. All vectors expressed a GFP bicistronically. A gate was set for GFP + cells and the cell death at different times as indicated was analyzed by flow cytometry. Numbers (mean \pm S.D.) indicate cell percentage of the gated cells. (b) The infected G1ME cells were further cultured for 2 days and stained with PI. A gate was set for GFP + cells and the cell cycle profile was analyzed by flow cytometry. The cell cycle profile was processed by FlowJo software. Table is the statistic of two independent experiments. Numbers (mean \pm S.D.) indicate cell percentage. (c) The infected G1ME cells were further cultured for 2 days and labeled with BrdU for 30 min. The resultant cells were stained with anti-BrdU-APC antibody and DAPI. A gate was set for GFP + cells and analyzed by flow cytometry. Numbers indicate percentage of the gated cells. Representative results from two independent experiments were shown. (d) The infected cells were also sorted 2 days after infection for preparing RNA. The expression of p21 and p27 were measured by quantitative RT-PCR. ${ }^{*}$ Indicates significance $(P<0.05)$ compared with control

Y410 and restored the suppressed ERK phosphorylation by PSTPIP2 (Figure 6b). In addition, we overexpressed a constitutively active form of LYN (LYN CA) in PSTPIP2 knockdown K562 cells. In contrast to LYN DN, the constitutively active form of LYN normalized CD41 expression upregulated by PSTPIP2 knockdown (Figure 6c). Correspondingly, ERK phosphorylation was repressed by LYN CA (Figure 6d). These results suggest that LYN likely mediates the effect of PSTPIP2 on megakaryocyte differentiation and signaling.

PSTPIP2 inhibits megakaryopoiesis in mouse bone marrow cells. To further verify the effect of PSTPIP2 on megakaryopoiesis, we overexpressed PSTPIP2 in primary lineage-depleted mouse bone marrow progenitor cells and measured megakaryocyte development. PSTPIP2 overexpression significantly reduced CD41 and CD42 expression as well as polyploidization of CD41 + cells (Figures $7 a-c$ ). Furthermore, it impaired CFU-GM (colony-forming unitgranulocyte/macrophage) and CFU-MK (colony-forming unit-megakaryocytic) (Figure 7d). These observations confirm that PSTPIP2 is a negative regulator of megakaryopoiesis in primary cells.

\section{Discussion}

TPO stimulation can potently activate MAPK/ERK signaling that is critical for megakaryocyte proliferation and differentiation. ${ }^{25-27}$ However, little is known about the regulation of TPOinduced MAPK/ERK activation. Previous studies showed that Src family kinases such as LYN and FYN were activated on TPO stimulation and in turn negatively regulated ERK activation in megakaryocytes. ${ }^{22,28,29}$ More recent research suggests that TPO-activated FAK may mediate TPO-induced Src family kinases activation. ${ }^{30}$ In this way, TPO-induced ERK activation may be constrained under physiological conditions. Considering that SHC binds to tyrosine sites of MPL and PEST family phosphatases, including PTP-PEST and PTPHSCF, the SHC may also recruit these phosphatases to the proximity of MPL. ${ }^{31,32}$ In addition, CSK is known to be tightly 

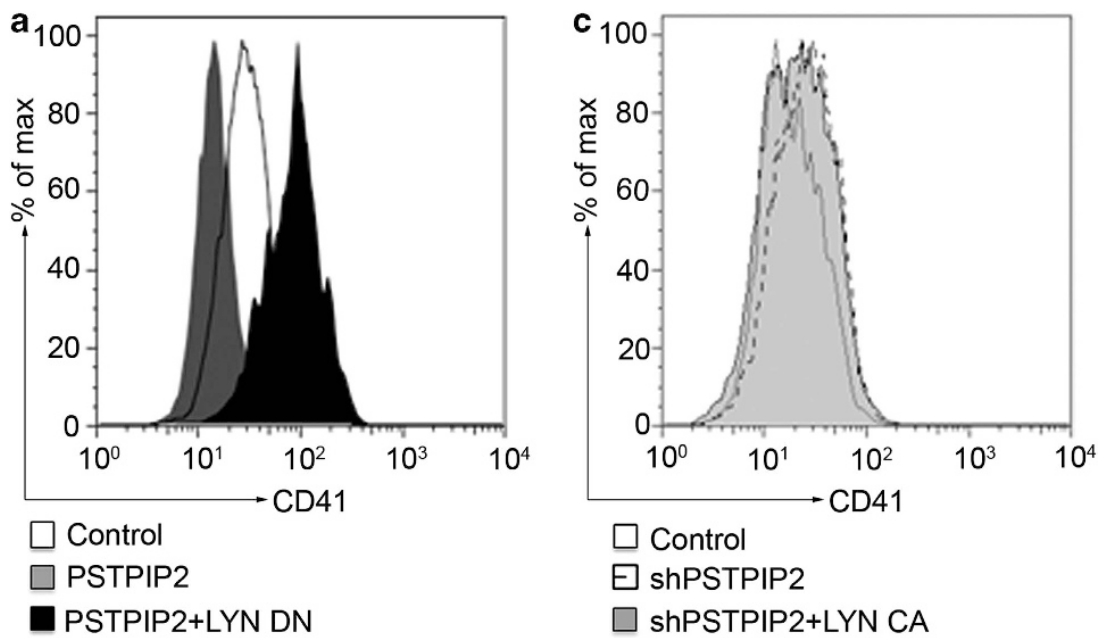

b

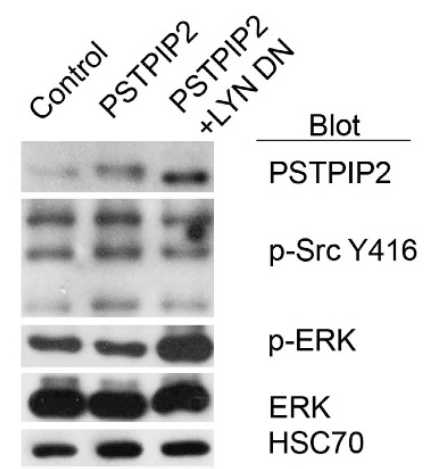

d

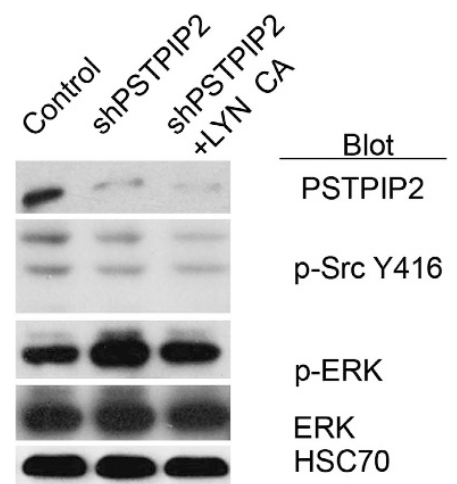

Figure 6 LYN mediates the effect of PSTPIP2 on megakaryocytic differentiation. (a) K562 were infected with control retrovirus vectors (Control, pMSCVpuro plus pMSCVneo empty vectors), PSTPIP2 (PSTPIP2-expressing vector plus pMSCVneo empty vector), or PSTPIP2 plus dominant negative form of LYN (PSTPIP2 + LYN DN) and selected with neomycin and puromycin. The selected cells were treated with TPA for 2 days and the expression of CD41 were measured by staining with anti-CD41-PE antibody and analyzed by flow cytometry. (b) The selected cells were also used for western blot for detecting the expression or phosphorylation of proteins as indicated. (c) K562 cells were infected with control retrovirus vectors (Control, pMSCVpuro plus pMSCVneo empty vectors), shPSTPIP2 (PSTPIP2-expressing vector plus pMSCVneo empty vector), or shPSTPIP2 plus constitutively active form of LYN (PSTPIP2 + LYN CA) and selected with neomycin and puromycin. The selected cells were treated with TPA for 2 days and the expression of CD41 were measured by staining with anti-CD41-PE antibody and analyzed by flow cytometry. (d) The selected cells were also used for western blot for detecting the expression or phosphorylation of proteins as indicated. All histograms and blots are representative results from two independent experiments with similar results

associated with PEST family phosphatase. Thus, $\mathrm{SHC}$ is very likely to bring CSK to the proximity of MPL and subsequently repress Src family kinases. ${ }^{21}$ Although the mechanism by which FAK is activated by TPO signaling remains unknown, recruitment of PEST family phosphatase/CSK may form antagonistic machinery to the activation of Src family kinases by FAK and secure TPO-induced MAPK/ERK activation. By this way, TPO signaling may balance the activation or repression of MAPK/ERK and finely tune megakaryocyte proliferation and differentiation.

Our study also suggests that GATA-1 participates in the fine-tuning MAPK/ERK activation in TPO signaling. GATA-1 has been shown to regulate TPO signaling as well as IFN- $\gamma$ signaling by maintaining JAK2 and STAT1 expression. ${ }^{10-12}$ In this study, we further showed that GATA-1 positively regulates MAPK/ERK activation by repressing negative regulator PSTPIP2. Upregulation of PSTPIP2 due to GATA-1 deficiency somehow inhibits CSK activity and disturbs the balance between FAK and CSK. Loss of inhibition by CSK leads to enhanced activation of Src family kinases and subsequently causes ERK inhibition. Thus, our study reveals a novel mechanism by which GATA-1 tunes TPO-induced ERK activation to promote megakaryocyte differentiation by inhibiting the negative regulator such as PSTPIP2, which represses PEST family phosphatase/CSK and activates Src family kinases. Our study also provides an example how extrinsic TPO signaling and intrinsic megakaryocyte/erythrocyte lineage-specific transcription factor may coordinate to regulate megakaryopoiesis.

How PSTPIP2 inhibits PEST family phosphatase/CSK remains unknown. One possibility is that PEST family phosphatase may dephosphorylate PSTPIP2 and may affect its binding to Src family kinases. However, we observed comparable LYN binding to the W232A mutant that abrogates its interaction with PEST family phosphatase. Most likely, the binding PSTPIP2 to the PEST phosphatase somehow affects CSK function. It has been shown that c-Src phosphorylation by CSK requires right position of the C-terminal tail of c-Src at the edge of the active site of CSK. ${ }^{33}$ It is possible that tight association of Src family kinases, PSTPIP2, and PEST family 
a
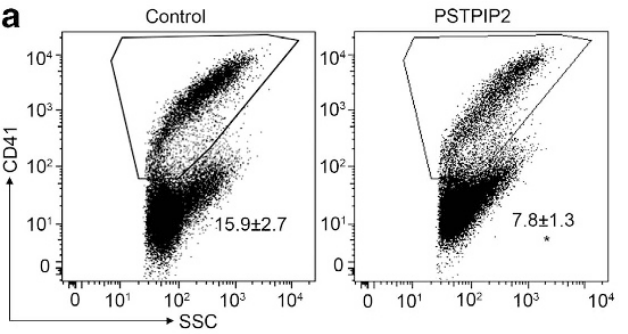

C

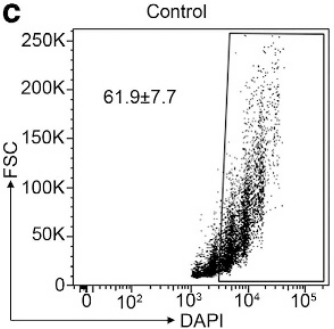

b
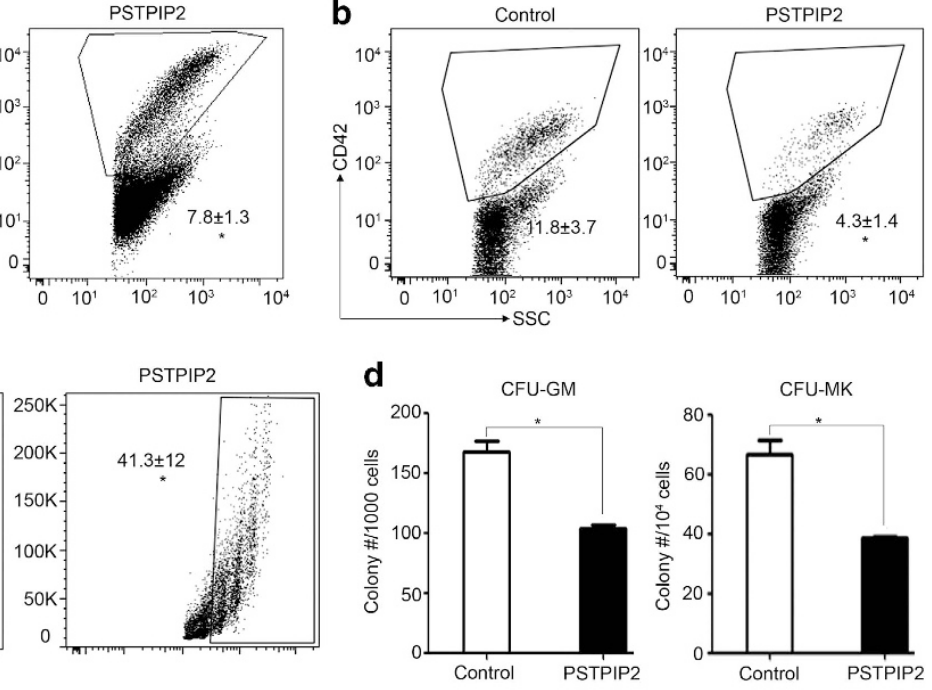

d
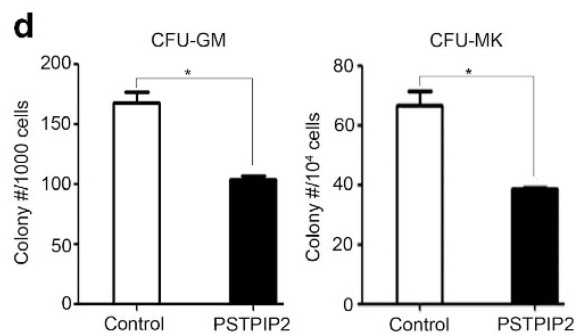

Figure 7 Ectopic expression of PSTPIP2 inhibits megakaryocytes development in primary mouse bone marrow cells. (a) Lineage-depleted hematopoietic stem/progenitor cells purified from mouse bone morrow were infected with control or PSTPIP2-expressing retrovirus, selected with puromycin, and further cultured in megakaryocyte differentiation medium for 3 days. The expression of CD41 in control or PSTPIP2-overexpressing cells was detected by staining cells with anti-CD41-APC antibody analyzed by flow cytometry. (b) The expression of CD42 in the resultant cells was measured by staining cells with anti-CD42-PE antibody. (c) The resultant cells were stained with antiCD41-PE and DAPI. The CD41 + cells were gated and further analyzed for DNA content. The gated events represented cells with DNA content greater than 4N. (d) The infected cells were also used for CFU-GM or CFU-Mk assay. All results are statistics of two independent experiments with duplicates. * Indicates significance $(P<0.05)$ compared with control

phosphatase/CSK may somehow lead to a steric obstacle for CSK and Src family kinases interaction that impairs Src family kinases phosphorylation by CSK.

It is interesting to notice that PSTPIP2 causes cell cycle arrest at $S$ phase and reduces CFU-GM and CFU-Mk in mouse bone marrow progenitors. This effect seems dependent on PEST family phosphatases. It is well known that MPL cause proliferation and differentiation through three major pathways: MAPK/ERK, JAK/STAT and PI3K/AKT. However, we failed to detect any significant changes in STAT3, STAT5 or AKT phosphorylation in PSTPIP2-overexpressing G1ME cells (Supplementary Figure S3). These results suggest that PSTPIP2 may inhibit TPO-induced proliferation through a novel mechanism. One possibility is that PSTPIP2 may cause cell proliferation inhibition through Src family kinase. Although Src family kinases have been known as oncogenes in various types of cancers, present evidence supports Src family kinases as negative regulators of megakaryocyte proliferation. For instance, LYN was activated by TPO signaling through TPO receptor tyrosine site Y599 and negatively regulated ERK activation. ${ }^{28}$ It also reduced phosphorylation of c-MPL without affecting STAT3, STAT5, or AKT phosphorylation. In addition, LYN-null mice display increased CFU-Mk, ${ }^{29}$ suggesting that $L Y N$ may repress megakaryocyte proliferation by inhibiting MAPK/ERK. Further supporting this idea, we observed reduced proliferation in K562 cells overexpressing LYN CA (data not shown) with reduced ERK phosphorylation. In addition, Pstpip2 was shown to inhibit macrophage proliferation. ${ }^{19}$ Pstpip2-deficient mice exhibited increased macrophage progenitors and CSF-1-induced hyperactivation of ERK1/2 and STAT1.Thus, it is very much possible that PSTPIP2 may inhibit bone marrow progenitor cell proliferation by activating Src family kinases, which subsequently leads to repression of ERK1/2. However, we cannot exclude the possibility that the PEST family phosphatases may contribute to megakaryocyte proliferation inhibition by PSTPIP2 through a Src family kinases or LYN-independent mechanism.

Taken together, we have identified PSTPIP2 as a novel negative regulator of megakaryopoiesis in part by modulating TPO signaling. In this way, GATA-1 can finely tune TPO signaling and coordinate megakaryopoiesis. Dysregulation of PSTPIP2 due to GATA-1 deficiency may contribute to abnormal proliferation and terminal differentiation in megakaryocytes.

\section{Materials and Methods}

Hematopoietic cells and megakaryocyte culture. Y10, CMK, and K562 cells were maintained in RIPM 1640 medium supplemented with $10 \%$ fetal bovine serum (Thermo Scientific HyClone, Logan, UT, USA) and penicillin/ streptomycin. G1ME cells were cultured in $\alpha$-MEM medium supplemented with $20 \%$ fetal bovine serum and 1\% TPO conditional medium as previously described. ${ }^{12}$ Primary mouse megakaryocytes were cultured in a serum-free system as described previously. ${ }^{12}$ C57BL/6 mice were maintained in microisolator housing within a barrier facility. All animal studies were approved by the Animal Care and Use Committees of Northwestern University and Wuhan University. For human megakaryocyte culture, $\mathrm{CD} 34^{+}$cells were grown in serum-free medium (IMDM supplemented with $100 \mathrm{U} / \mathrm{ml}$ penicillin/streptomycin, $2 \mathrm{mM}$ glutamine, $76 \mathrm{nM} \alpha$-monothioglycerol, 1.5\% BSA, $20 \mu \mathrm{g} / \mathrm{ml}$ lipids, insulin-transferrin-selenium solution (Cat\#41400045, Life Technologies), and $10 \mathrm{ng} / \mathrm{ml}$ recombinant human thrombopoietin). Cells were cultured at $37^{\circ} \mathrm{C}$ with $5 \% \mathrm{CO}_{2}$ and collected at different time point.

Luciferase assay. Pstpip2 gene promoter report construct was made by subcloning a $1-\mathrm{kb}$ fragment of its promoter upstream region into $\mathrm{pGL} 3$ Basic vector (termed as P2-pro). A second vector was made by subcloning the 265-bp core region of the putative GATA-1-binding site between the P2-pro and luciferase (termed as P2-pro/G1BS). All luciferase reporter constructs expressed firefly luciferase and assays were performed in 293T cells. Briefly, P2-pro or P2-pro/ G1BS cotransfected with the same amount of pRL-TK (internal control) expressing 
a renila luciferase in combination with other plasmids as indicated. The dual luciferase activity was assayed according to the manufacturer's instructions (dual-luciferase reporter assay system, Promega, Madison, WI, USA). The firefly luciferase activity was normalized by renila luciferase activity inner control. The activity of each promoter was further normalized by the control group and presented as relative luciferase activity.

Retroviral and lentiviral transduction. For overexpression of PSTPIP2, retrovirus (pMIGR1 or pMSCV vector) or lentivirus (pHAGE vector) systems were used. For downregulation of PSTPIP2, pKLO.1 lentivirus system was used. The retroviral or lentiviral stocks were prepared as previously described and used for infection of K562, G1ME, or primary mouse bone marrow progenitor cells. ${ }^{12}$ The human PSTPIP2 cDNA was cloned by RT-PCR from K562 cell with primers: forward $5^{\prime}$-CCACCATGACGCGCTCACTG- $3^{\prime}$ and reverse $5^{\prime}$-TTACTGATAGAGC AAACTGT-3'. For PSTPIP2 knockdown, we used two hairpin sequences (TRCN0000003019 and TRCN0000003020) from Public TRC Portal (http://www. broadinstitute.org/rnai/public/gene/details?geneld=TRCG0000000608).

CHIP assay. ChIP assays were performed by using an antibody specifically against GATA-1 (Cat\# SC-1234, Santa Cruz Biotechnology, Santa Cruz, CA, USA) or a normal Goat IgG as previously described. ${ }^{34}$ The chromatin DNA enrichment in intron 1 region of Pstpip2 gene or control region of Gapdh gene was measured by quantitative PCR. Relative occupancy was determined by comparing the signals with a dilution series of the input sample amplified with the same primers. Gapdh served as negative control gene that was not regulated by GATA-1. Primer sequences are available on request.

Cell cycle profiling. Cell cycle profile was assayed by staining with or propidium iodide (PI) or BrdU incorporation as described before. ${ }^{34}$ For $\mathrm{PI}$ staining, control or PSTPIP2-overexpression G1ME cells were fixed, permeablized, and stained with PI $(1 \mu \mathrm{g} / \mathrm{ml})$. Data were acquired by Flow Cytometry and cell cycle profile was processed by FlowJo software (Tree Star Inc, Ashland, OR, USA). For BrdU incorporation, cells were labeled with bromodeoxyuridine (BrdU, $3 \mathrm{mg} / \mathrm{ml}$ ) for half hour, fixed, permeablized, and stained with Alexa 647-labeled anti-BrdU antibody and 4', 6-diamidino-2-phenylindole (DAPI, $1 \mu \mathrm{g} / \mathrm{ml})$. Flow cytometry was performed on an LSRII apparatus or Calibur (BD Biosciences, San Jose, CA, USA) and data were analyzed by FlowJo software.

Colony-forming unit assays. The lineage-negative bone marrow were transduced with retrovirus and selected with puromycin $(1 \mu \mathrm{g} / \mathrm{ml})$ for 1 day after infection. The CFU-GM and CFU-Mk were performed as previously described. ${ }^{12}$

RNA preparation and quantitative RT-PCR. Total RNA was extracted with Trizol and CDNA was prepared with reverse transcriptase by standard procedure following the instruction (Life Technologies). Quantitative PCR was performed under the following conditions: hot start at $95^{\circ} \mathrm{C}$ for $15 \mathrm{~min}$ followed by $95^{\circ} \mathrm{C}$ for $30 \mathrm{~s}, 63^{\circ} \mathrm{C}$ for $30 \mathrm{~s}$, and $72^{\circ} \mathrm{C}$ for $30 \mathrm{~s}$ for 40 cycles. The relative quantities of real-time PCR products were determined using the comparative $\Delta \Delta C T$ method. Primer sequences are available upon request.

Flow cytometry. Mouse megakaryocyte differentiation was analyzed by staining cell surface markers with fluorescence labeled anti-CD41 (cat no. 17-0411, eBioscience, San Diego, CA, USA) or anti-CD42 (cat no. 12-0421-82, eBioscience) antibodies or staining DNA with 4,6-diamidinio-2-phenylindole (DAPI, $1 \mu \mathrm{g} / \mathrm{ml}$ ) or propidium iodide (PI, $1 \mu \mathrm{g} / \mathrm{ml})$. For cell death, cells were stained with PE-labeled anti-Annexin V antibody (cat no. 1014-200, Biovision, Milpitas, CA, USA) in staining buffer (10 mM HEPES, pH 7.4; $140 \mathrm{mM} \mathrm{NaCl} ; 2.5 \mathrm{mM} \mathrm{CaCl}_{2}$ ) and analyzed by flow cytometry. All histogram analyses were representative photos from at least two experiments with similar results. Dot plot analyses were statistics (mean \pm S.D.) of two experiments with duplicates.

Immunoprecipitation and western blot. Briefly, $10 \times 10^{6}$ cells were

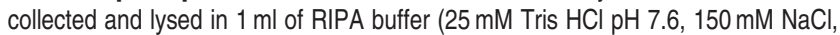
$1 \%$ NP- $40,1 \%$ sodium deoxycholate, $0.1 \%$ SDS) for immunoprecipitation. Cell extracts were pre-cleared by mixing lysates with $2 \mu \mathrm{g}$ of normal control $\lg \mathrm{G}$ and $50 \mu \mathrm{l}$ of protein $\mathrm{G}$ agarose and incubating for $4 \mathrm{~h}$ at $4{ }^{\circ} \mathrm{C}$. The pre-cleared lysates were mixed with $2 \mu \mathrm{g}$ of the appropriate antibodies plus $20 \mu \mathrm{l}$ of protein $\mathrm{G}$ agarose beads and rotated overnight at $4{ }^{\circ} \mathrm{C}$. The immunoprecipitated complexes were obtained by centrifugation, washed three times with RIPA lysis buffer and boiled at $75^{\circ} \mathrm{C}$ in loading buffer for $10 \mathrm{~min}$ for western blot. Cell lysates were also directly used for western blot analysis. All western blot photos were representative photos from at least two experiments with similar results. Antibodies for HSC 70 (cat no. SC-7298), Lyn (cat no. SC-7274), Fyn (cat no. SC-16), PSTPIP2 (cat no. SC-83015), GATA1 (cat no. SC-1234), SHC (cat no. SC-1695) were purchased from Santa Cruz Biotechnology. Antibody for FLAG (cat no. F3165) was obtained from Sigma. Antibodies for ERK (cat no. 4695), p-ERK (cat no. 4370), p-SRC (cat no. 2101) were purchased from Cell Signaling Technology.

Statistics. All statistical analyses were performed using the Student's $t$-test (2 tailed, unpaired). A $P$-value of 0.05 or less was considered significant.

\section{Conflict of Interest}

The authors declare no conflict of interest.

Acknowledgements. We thank Xingtong Liu for constructing expression plasmids. This work was supported by National Natural Science Foundation of China (Grant nos 81070406 and 31371481 to Z Huang; Grant no. 81000201 to L Li), PhD Programs Foundation of Ministry of Education of China (Grant No. 20110141110016 to Z Huang), and New Century Excellent Talents in University (NCET) of Ministry of Education of China (NCET-12-0422 to Z Huang).

1. Gurney AL, Carver-Moore K, de Sauvage FJ, Moore MW. Thrombocytopenia in c-mpl-deficient mice. Science 1994; 265: 1445-1447.

2. de Sauvage FJ, Carver-Moore K, Luoh SM, Ryan A, Dowd M, Eaton DL et al. Physiological regulation of early and late stages of megakaryocytopoiesis by thrombopoietin. J Exp Med 1996; 183: 651-656.

3. Fujiwara Y, Browne CP, Cunniff K, Goff SC, Orkin SH. Arrested development of embryonic red cell precursors in mouse embryos lacking transcription factor GATA-1. Proc Natl Acad Sci USA 1996; 93: 12355-12358.

4. Pevny L, Lin CS, D'Agati V, Simon MC, Orkin SH, Costantini F. Development of hematopoietic cells lacking transcription factor GATA-1. Development 1995; 121: 163-172.

5. Pevny L, Simon MC, Robertson E, Klein WH, Tsai SF, D'Agati V et al. Erythroid differentiation in chimaeric mice blocked by a targeted mutation in the gene for transcription factor GATA-1. Nature 1991; 349: 257-260.

6. Shivdasani RA, Fujiwara $Y, M c D e v i t t ~ M A$, Orkin SH. A lineage-selective knockout establishes the critical role of transcription factor GATA-1 in megakaryocyte growth and platelet development. EMBO J 1997; 16: 3965-3973.

7. Wechsler J, Greene M, McDevitt MA, Anastasi J, Karp JE, Le Beau MM et al. Acquired mutations in GATA1 in the megakaryoblastic leukemia of Down syndrome. Nat Genet 2002; 32: 148-152.

8. Mundschau G, Gurbuxani S, Gamis AS, Greene ME, Arceci RJ, Crispino JD. Mutagenesis of GATA1 is an initiating event in Down syndrome leukemogenesis. Blood 2003; 101: $4298-4300$.

9. Li Z, Godinho FJ, Klusmann JH, Garriga-Canut M, Yu C, Orkin SH. Developmental stageselective effect of somatically mutated leukemogenic transcription factor GATA1. Nat Genet 2005; 37: 613-619.

10. Chou ST, Khandros E, Bailey LC, Nichols KE, Vakoc CR, Yao Y et al. Graded repression of PU.1/Sfpi1 gene transcription by GATA factors regulates hematopoietic cell fate. Blood 2009; 114: 983-994.

11. Muntean AG, Crispino JD. Differential requirements for the activation domain and FOG-interaction surface of GATA-1 in megakaryocyte gene expression and development. Blood 2005; 106: 1223-1231.

12. Huang Z, Richmond TD, Muntean AG, Barber DL, Weiss MJ, Crispino JD. STAT1 promotes megakaryopoiesis downstream of GATA-1 in mice. J Clin Invest 2007; 117: 3890-3899.

13. Chen E, Beer PA, Godfrey AL, Ortmann CA, Li J, Costa-Pereira AP et al. Distinct clinical phenotypes associated with JAK2V617F reflect differential STAT1 signaling. Cancer Cell 2010; 18: 524-535.

14. Dore LC, Chlon TM, Brown CD, White KP, Crispino JD. Chromatin occupancy analysis reveals genome-wide GATA factor switching during hematopoiesis. Blood 2012; 119: 3724-3733.

15. Chlon TM, Dore LC, Crispino JD. Cofactor-mediated restriction of GATA-1 chromatin occupancy coordinates lineage-specific gene expression. Mol Cell 2012; 47: 608-621.

16. Yu M, Riva L, Xie H, Schindler Y, Moran TB, Cheng Y et al. Insights into GATA-1-mediated gene activation versus repression via genome-wide chromatin occupancy analysis. Mol Cell 2009; 36: 682-695.

17. Yeung YG, Soldera S, Stanley ER. A novel macrophage actin-associated protein (MAYP) is tyrosine-phosphorylated following colony stimulating factor-1 stimulation. $\mathrm{J}$ Biol Chem 1998; 273: 30638-30642. 
18. Wu Y, Dowbenko D, Lasky LA. PSTPIP 2, a second tyrosine phosphorylated cytoskeletal-associated protein that binds a PEST-type protein-tyrosine phosphatase. J Biol Chem 1998; 273: 30487-30496.

19. Chitu V, Ferguson PJ, de Bruijn R, Schlueter AJ, Ochoa LA, Waldschmidt TJ et al. Primed innate immunity leads to autoinflammatory disease in PSTPIP2-deficient $\mathrm{cmo}$ mice. Blood 2009; 114: 2497-2505.

20. Grosse J, Chitu V, Marquardt A, Hanke P, Schmittwolf C, Zeitlmann L et al. Mutation of mouse Mayp/Pstpip2 causes a macrophage autoinflammatory disease. Blood 2006; 107 3350-3358.

21. Veillette A, Rhee I, Souza CM, Davidson D. PEST family phosphatases in immunity, autoimmunity, and autoinflammatory disorders. Immunol Rev 2009; 228: 312-324.

22. Lannutti BJ, Shim MH, Blake N, Reems JA, Drachman JG. Identification and activation of Src family kinases in primary megakaryocytes. Exp Hematol 2003; 31: 1268-1274.

23. Davidson D, Cloutier JF, Gregorieff A, Veillette A. Inhibitory tyrosine protein kinase p50csk is associated with protein-tyrosine phosphatase PTP-PEST in hemopoietic and non-hemopoietic cells. J Biol Chem 1997; 272: 23455-23462.

24. Stachura DL, Chou ST, Weiss MJ. Early block to erythromegakaryocytic development conferred by loss of transcription factor GATA-1. Blood 2006; 107: 87-97.

25. Rouyez MC, Boucheron C, Gisselbrecht S, Dusanter-Fourt I, Porteu F. Control of thrombopoietin-induced megakaryocytic differentiation by the mitogen-activated protein kinase pathway. Mol Cell Biol 1997; 17: 4991-5000.

26. Rojnuckarin P, Drachman JG, Kaushansky K. Thrombopoietin-induced activation of the mitogen-activated protein kinase (MAPK) pathway in normal megakaryocytes: role in endomitosis. Blood 1999; 94: 1273-1282.

27. Melemed AS, Ryder JW, Vik TA. Activation of the mitogen-activated protein kinase pathway is involved in and sufficient for megakaryocytic differentiation of CMK cells. Blood 1997; 90: 3462-3470.
28. Lannutti BJ, Drachman JG. Lyn tyrosine kinase regulates thrombopoietin-induced proliferation of hematopoietic cell lines and primary megakaryocytic progenitors. Blood 2004; 103: 3736-3743.

29. Lannutti BJ, Minear J, Blake N, Drachman JG. Increased megakaryocytopoiesis in Lyn-deficient mice. Oncogene 2006; 25: 3316-3324.

30. Hitchcock IS, Fox NE, Prevost N, Sear K, Shattil SJ, Kaushansky K. Roles of focal adhesion kinase (FAK) in megakaryopoiesis and platelet function: studies using a megakaryocyte lineage specific FAK knockout. Blood 2008; 111: 596-604.

31. Alexander WS, Maurer AB, Novak U, Harrison-Smith M. Tyrosine-599 of the c-Mpl recepto is required for Shc phosphorylation and the induction of cellular differentiation. EMBO $J$ 1996; 15: 6531-6540.

32. Drachman JG, Kaushansky K. Dissecting the thrombopoietin receptor: functional elements of the Mpl cytoplasmic domain. Proc Natl Acad Sci USA 1997; 94: 2350-2355.

33. Levinson NM, Seeliger MA, Cole PA, Kuriyan J. Structural basis for the recognition of c-Src by its inactivator Csk. Cell 2008; 134: 124-134.

34. Huang Z, Dore LC, Li Z, Orkin SH, Feng G, Lin S et al. GATA-2 reinforces megakaryocyte development in the absence of GATA-1. Mol Cell Biol 2009; 29: 5168-5180.

(c) (i) (2)(2) Cell Death and Disease is an open-access journal published by Nature Publishing Group. This work is licensed under a Creative Commons Attribution-NonCommercialShareAlike 3.0 Unported License. To view a copy of this license, visit http://creativecommons.org/licenses/by-nc-sa/3.0/

Supplementary Information accompanies this paper on Cell Death and Disease website (http://www.nature.com/cddis) 